\title{
Sleep-disordered breathing-related neurocognitive impairment, time to think beyond hypoxia and sleep fragmentation?
}

\author{
David Wang • Brendon J. Yee • Luke Rowsell
}

Received: 14 May 2014 / Accepted: 19 May 2014 / Published online: 4 June 2014

(C) Springer-Verlag Berlin Heidelberg 2014

It has been well accepted that sleep-disordered breathing (SDB) is associated with impaired neurocognitive and behavioral performance and may consequently cause motor vehicle accidents and occupational injuries [1-3]. However, the relevant pathophysiological mechanism causing this is unclear. In particular, the key determinants of the neurocognitive/ behavioral impairment in SDB are not known. Many candidate factors such as hypoxia, sleep fragmentation, apneahypopnea index (AHI), BMI, sleep time, and metabolic factors have been studied, but none show a strong correlation with neurocognitive/behavioral impairment [4, 5]. Of these factors, intermittent hypoxia and sleep fragmentation has the strongest evidence and may play a more important role than other factors [4-6]. However, lack of robust findings means that it is difficult to claim these two parameters as "key determinants". In a landmark US study in this field (APPLES study), Quan et al. tested long-term neurocognitive outcomes of continuous positive airway pressure (CPAP) on 1,204 obstructive sleep apnea (OSA) patients, using a robust randomized, double-blind, and sham-controlled design [7]. They found that severity of $\mathrm{O}_{2}$ desaturation was the strongest predictor, but explaining only $<2 \%$ of the variance of neurocognitive performance. Sleep fragmentation was not a significant predictor [7]. In a multicenter European study,

D. Wang $(\varangle) \cdot$ B. J. Yee

Department of Respiratory and Sleep Medicine, Royal Prince Alfred Hospital, Missenden Rd, Camperdown, NSW 2050, Australia e-mail: david.wang@sydney.edu.au

D. Wang $\cdot$ B. J. Yee $\cdot$ L. Rowsell

Woolcock Institute of Medical Research, University of Sydney, Glebe Point Rd, Glebe, NSW, Australia

B. J. Yee $\cdot$ L. Rowsell

Centre for Integrated Research and Understanding of Sleep (CIRUS),

University of Sydney, Glebe, NSW, Australia
1,649 OSA patients with excessive daytime sleepiness (EDS) were compared with 1,233 OSA patients without EDS [8]. The two groups were found to have only $1 \%$ difference in $\mathrm{SpO}_{2}$ nadir, no difference in mean $\mathrm{SpO}_{2}$, and only four events per hour difference in Arousal index $(37 / \mathrm{h}$ in EDS and 33/h in non-EDS group) [8].

Given the above context, it is not surprising that in the current issue of the journal, Olaithe et al. found that neither hypoxia nor sleep fragmentation significantly predict cognitive performances in any domain [9]. The authors thoroughly assessed different domains of cognitive function and overnight polysomnography (PSG) in 150 Australians ranging from no-OSA to severe OSA. They examined the potential relationships between hypoxia/sleep fragmentation and cognitive function using structured equation modeling and carefully controlled for the confounding factors of IQ and age. They found that hypoxia was unrelated to any cognitive measures. Although modest relationships between sleep fragmentation and certain domains of cognition were found, the significance was lost after controlling for age [9]. Indeed, given that daytime cognition is affected by so many coexisting factors, any claim of relationship without a rigorous control of confounding factors would be misleading. From this aspect, the well-controlled study from Olaithe et al. can certainly add weight in the literature [9].

Importantly, data from Olaithe et al. and other studies may imply that we should not limit our thoughts solely to hypoxia and sleep fragmentation when considering the mechanisms of SDB-related cognitive impairment. Recent data have suggested other lines of investigation. For example, sleep fragmentation is usually quantified by arousal index from overnight EEG/PSG. Recent data suggest that certain parameters from EEG spectral analyses are related to daytime neurocognitive/behavioral function [10, 11]. Quantitative individual EEG band power/percentage or certain ratios showing overall slowing of EEG (such as Delta/Alpha ratio, Delta+ 
Theta/Alpha+Beta ratio) or detrended fluctuation analysis (DFA) could be considered [10, 11]. In addition, while SDB is characterized by recurrent episodes of both hypoxia and hypercapnia, the relationship of hypercapnia and daytime sleepiness has been rarely investigated [11]. This omission is likely due to the lack of clinical equipment to reliably measure $\mathrm{paCO}_{2}$ during sleep. We recently observed that hypercapnic SDB patients $(n=97)$ had paradoxically high percentage of slow wave sleep; awake $\mathrm{pCO}_{2}$ measured by $\mathrm{ABG}$ was the best predictor for the slowing of EEG [12]. There was a crosscorrelation between a reduced wake $\mathrm{paCO}_{2}$, a faster sleep EEG (reduced Delta/Alpha ratio) and reduced daytime sleepiness during positive airway pressure treatment in hypercapnic SDB patients [11]. Multiple regression analyses showed that the degree of change in hypercapnia but not hypoxia was the only significant predictor of both the Delta/Alpha ratio and daytime sleepiness [11]. In healthy subjects, there is also a positive linear relationship between the increasing hypercapnia and the slowing of EEG (increased Delta/Alpha ratio), while hypoxia did not show such an effect [13]. While preliminary, these studies raise the possibility that hypercapnia may be mechanistically more important than hypoxia in SDBrelated neurocognitive/behavioral impairment $[12,11,13]$.

The search for clinical markers to predict those vulnerable to neurobiological impairment from SDB is important for disease risk stratification and treatment prioritization. It is clear that current conventional information obtained from a sleep study does not provide such information. New biomarkers of this vulnerability remain a challenge for our field.

Funding Dr. David Wang is supported by NHMRC Project Grant (No. 1043633). Luke Rowsell is supported by CIRUS.

\section{References}

1. Lindberg E, Carter N, Gislason T, Janson C (2001) Role of snoring and daytime sleepiness in occupational accidents. Am J Respir Crit Care Med 164(11):2031-2035
2. Malhotra A, White DP (2002) Obstructive sleep apnoea. Lancet 360(9328):237-245. doi:10.1016/S0140-6736(02)09464-3

3. Teran-Santos J, Jimenez-Gomez A, Cordero-Guevara J (1999) The association between sleep apnea and the risk of traffic accidents. Cooperative Group Burgos-Santander. N Engl J Med 340(11):847851. doi:10.1056/NEJM199903183401104

4. Dempsey JA, Veasey SC, Morgan BJ, O'Donnell CP (2010) Pathophysiology of sleep apnea. Physiol Rev 90(1):47-112. doi:10. 1152/physrev.00043.2008

5. Engleman HM, Douglas NJ (2004) Sleep. 4: sleepiness, cognitive function, and quality of life in obstructive sleep apnoea/hypopnoea syndrome. Thorax 59(7):618-622

6. Beebe DW, Gozal D (2002) Obstructive sleep apnea and the prefrontal cortex: towards a comprehensive model linking nocturnal upper airway obstruction to daytime cognitive and behavioral deficits. J Sleep Res 11(1):1-16

7. Quan SF, Chan CS, Dement WC, Gevins A, Goodwin JL, Gottlieb DJ, Green S, Guilleminault C, Hirshkowitz M, Hyde PR, Kay GG, Leary EB, Nichols DA, Schweitzer PK, Simon RD, Walsh JK, Kushida CA (2011) The association between obstructive sleep apnea and neurocognitive performance - the Apnea Positive Pressure Long-term Efficacy Study (APPLES). Sleep 34(3):303-314B

8. Roure N, Gomez S, Mediano O, Duran J, Pena Mde L, Capote F, Teran J, Masa JF, Alonso ML, Corral J, Sanchez-Armengod A, Martinez C, Barcelo A, Gozal D, Marin JM, Barbe F (2008) Daytime sleepiness and polysomnography in obstructive sleep apnea patients. Sleep Med 9(7):727-731. doi:10.1016/j.sleep.2008.02.006

9. Olaithe M, Skinner T, Hillman D, Eastwood P, Bucks RS (2014) Cognition and nocturnal disturbance in OSA: importance of accounting for age and premorbid intelligience. Sleep Breath. doi:10.1007/ s11325-014-1000-2

10. D'Rozario AL, Kim JW, Wong KK, Bartlett DJ, Marshall NS, Dijk DJ, Robinson PA, Grunstein RR (2013) A new EEG biomarker of neurobehavioural impairment and sleepiness in sleep apnea patients and controls during extended wakefulness. Clin Neurophysiol 124(8):1605-1614. doi:10.1016/j.clinph.2013.02.022

11. Wang D, Piper AJ, Yee BJ, Wong KK, Kim JW, D’Rozario AL, Rowsell L, Dijk DJ, Grunstein RR (2014) Hypercapnia is a key correlate of EEG activation and daytime sleepiness in hypercapnic sleep-disordered breathing patients. J Clin Sleep Med 10(5):517-522. doi: $10.5664 / \mathrm{jcsm} .3700$

12. Wang D, Piper AJ, Wong KK, Yee BJ, Marshall NS, Dijk DJ, Grunstein RR (2011) Slow wave sleep in patients with respiratory failure. Sleep Med 12:378-383. doi:10.1016/j.sleep.2011.01.007

13. Wang D, Yee BJ, Wong KK, Kim JW, Dijk DJ, Duffin J, Grunstein RR (2014) Comparing the effect of hypercapnia and hypoxia on the electroencephalogram during wakefulness. Clin Neurophysiol. doi: 10.1016/j.clinph.2014.04.012 